\title{
A Novel Amplification-free SARS-CoV-2 Point-of-care Nucleic Acid Detection System based on Hybrid Capture Fluorescence Immunoassay
}

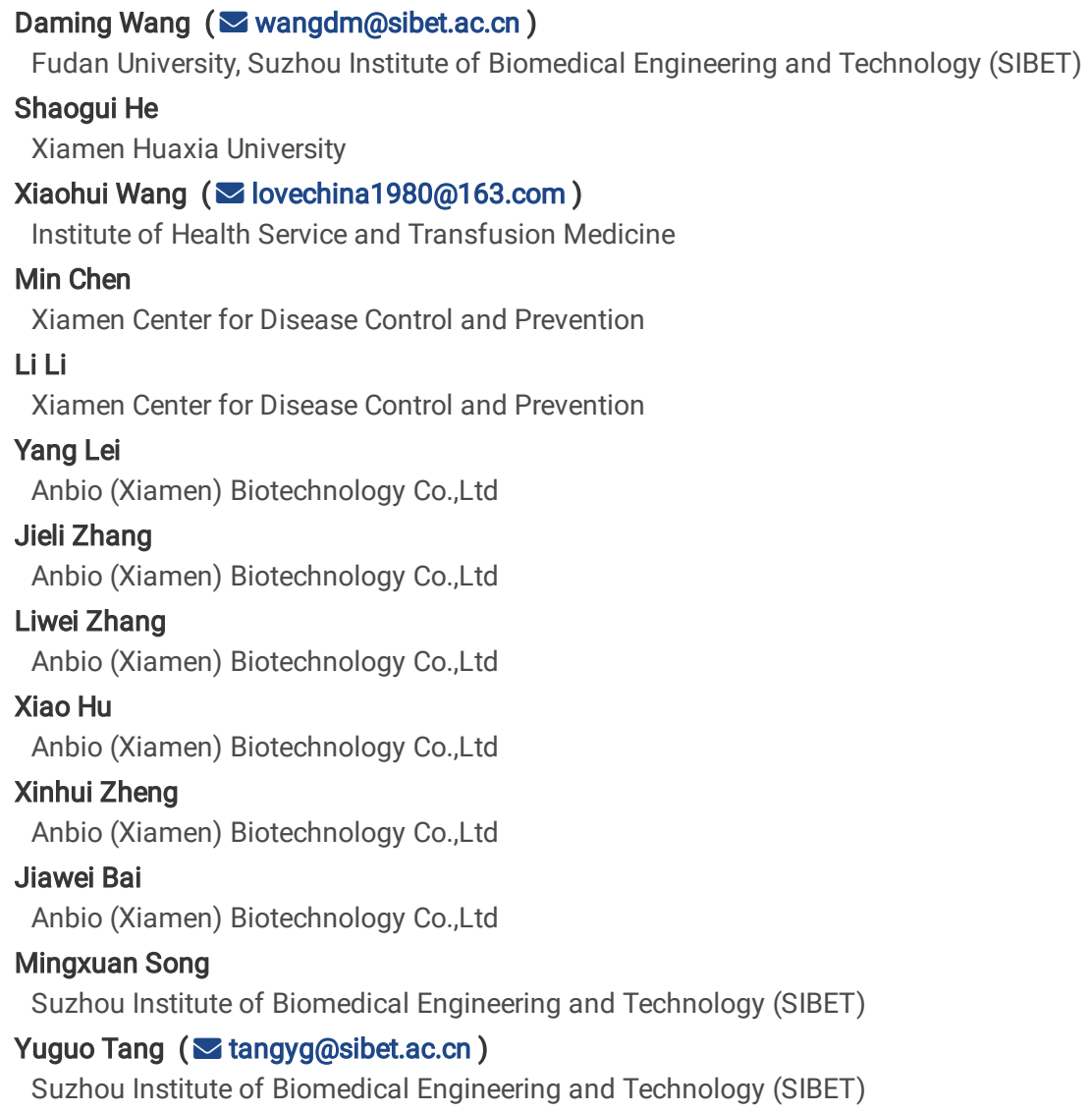




\section{Abstract}

Herein we presented a novel, rapid and amplification-free SARS-CoV-2 nucleic acid detection system based on hybrid capture fluorescence immunoassay (HC-FIA) technology. The usage of the monoclonal antibody S9.6 in recognizing DNA-RNA double-stranded hybrids enabled the conversion of nucleic acid testing into immunofluorescence carrying on a simple lateral flow dipstick. The established HC-FIA also exhibited satisfactory sensitivity, specificity and great robustness. The clinical evaluation of HC-FIA kit and fluorescence reading device are further processed in three hospitals independently. The results of 734 samples from 670 subjects indicated high consistency between our HC-FIA and quantitative polymerase chain reaction based commercially available kit or clinical diagnosis according to Kappa statistics. Altogether, HC-FIA related method and commercial test kit show unparalleled advantages as time saving, amplification-free, high throughput and portable POCT molecular diagnosis, which facilitates its application as on-site Severe acute respiratory syndrome coronavirus 2 (SARS-CoV-2) nucleic acid detection in epidemic prevention and control worldwide, especially during the outbreak.

\section{Introduction}

The novel coronavirus continued to spread globally, sweeping more than 100 countries and regions, and becoming a new threat to the Community of Shared Future for Mankind. The enveloped novel coronavirus, which was defined as SARS-CoV-2 by the International Committee on Taxonomy of Viruse (ICTV), belonged to the novel coronavirus of the genus $\beta$, with particles round or oval and a diameter ranging from 60 to $140 \mathrm{~nm}$. On January 11 th, 2020 , scientists published the whole genome sequence of the novel coronavirus on the Virology Organization website (virological.org) for the first time. Two weeks later, after being approved by National Medical Products Administration (NMPA) in case of emergency, four new crown virus nucleic acid detection assay kits were launched into the market. Its genetic characteristics were significantly different from Severe Acute Respiratory Syndrome related Coronavirus (SARSr-CoV) and Middle East Respiratory Syndrome related Coronavirus (MERSr-CoV). Current research showed that it had more than $87.5 \%$ homology with bat SARS-like coronavirus (bat-SL-CoVZC45 and bat-SL-CoVZXC21) [1].

To discriminate the confirmed Corona Virus Disease 2019 (COVID-19) cases from the suspected ones, the etiological diagnosis of the novel coronavirus pneumonia mainly relied on molecular biological methods such as nucleic acid detection and gene sequencing. Second-generation sequencing technology not only required sophisticated equipment, skilled operators but was time and cost consuming. By contrast, nucleic acid detection methods may more easily meet clinical needs for diagnosis by increasing productivity. Although the reverse transcription-based fluorescence quantitative PCR method exhibited high sensitivity and specificity, the turn around time (TAT) was still long due to the nucleic acid purification and amplification steps. If the aerosol of new coronavirus polluted the detection laboratory, the long-term unavailability of laboratory brought great difficulties to clinical diagnosis, especially during the outbreak of COVID-19. According to the latest report of Nature, nearly $60 \%$ of people with the novel coronavirus infection were with mild or no symptoms [2]. Therefore, there was an urgent need for the development of a fast, simple and high-throughput nucleic acid detection to solve the problem that reverse transcription-quantitative Polymerase Chain Reaction (RT-qPCR) might not have the ability to deal well with the serious challenge for global public health security.

The application of S9.6 monoclonal antibody with high affinity and selectivity to DNA-RNA hybrid molecules [3] was the basis for the design of our HC-FIA method in this study. This monoclonal antibody was raised to a DNA-RNA heteropolymers duplex prepared by transcription of $\varphi$ X174 single-stranded DNA with DNA-dependent RNA polymerase [3]. It only bound to DNA-RNA hybrids and had no response to single-stranded DNA, double-stranded DNA, doublestranded RNA, and ribosomal RNA. The capture of DNA-RNA hybrids by this monoclonal antibody did not have sequence specificity, which was widely used in immunoprecipitation experiments of R-loop and DNA-RNA double-stranded hybrids [4-6]. Commercially available S9.6 antibodies had been used in human papilloma virus (HPV) diagnostic reagents. The second-generation of hybrid capture technology (HC2) approved by Food and Drug Administration (FDA) can detect 13 high-risk HPV subtypes simultaneously. The monoclonal antibody was also used to capture the complexes formed by hybridization of small RNA and microRNA with various types of labeled DNA probes for signal conversion and amplification [7-11].

In this work, S9.6 monoclonal antibody-labeled Europium chelate-based fluorescent nanoparticles (FNPs) were employed to capture the hybridized doublestrands formed by the designed DNA probes and the RNA of SARS-CoV-2, and then performed immunofluorescence lateral flow dipstick by reading the accumulation of fluorescence signal. This detection platform was documented to be skillfully constructed with no requirements of amplification, and in line with the concept of point-of-care technology (POCT). It was expected to realize the molecular diagnosis in a "suitcase laboratory" to confirm the novel coronavirus infection and would play a critical role in control of its global epidemics. Moreover, being supported by a great number of clinical data, the HCFIA presented herein was not only a proof-of-concept research, but also had been developed into a commercial test kit for the diagnosis of SARS-CoV-2, which recently approved by NMPA and acquired the CE certification.

\section{Results}

\section{The principle of the biosensor HC-FIA}

In the novel HC-FIA detection system, probe DNA-functionalized FNPs were used to amplify the signal of virus RNA and DNA hybridizing. With the help of monoclonal antibody S9.6, the nucleic acid detection of virus RNA was transformed into an immunoassay through the sophisticated design. This type of antibody specifically bond with double helix structure of RNA-DNA hybrid and such recognition depended little on base sequence [3]. The design principle of HC-FIA biosensor was shown in Figure 1. The murine antibody S9.6 (Ab1) was pre-fixed on the test line (T) of the lateral flow dipstick, and the control line (C) was coated with goat anti-rabbit IgG polyclonal antibody. Pre-coated FNPs labeled by anti-DNA-RNA S9.6 (Ab2) and rabbit IgG antibody were firstly contained in the reaction tube. 
In the detection process, firstly, the SARS-CoV-2 in the throat swab or sputum sample was lysed and released, and the released RNA hybridized with the specific SARS-CoV-2 DNA probe. The resulting RNA-DNA hybrid was captured by S9.6 antibody (Ab2) labeled FNPs, and the complex flowed along the sample pad and the nitrocellulose membrane toward the absorbent paper under capillary forces. When passing through $T$ area, it would be captured by the coated mouse anti-DNA-RNA monoclonal antibody (Ab1) to form an "Ab1-RNA-DNA hybrid-fluorescence labeled Ab2" complex and a fluorescent signal gradually generated. In the case of $\mathrm{C}$ area, FNPs labeled rabbit IgG antibody in the reaction tube and the pre-fixed goat anti-rabbit IgG polyclonal antibody bound to form a "goat anti-rabbit IgG polyclonal antibody-FNPs labeled rabbit IgG antibody" (Figure 1A).

The fluorescence signal of the nanoparticles emitted by the excitation light was received by the fluorescence analysis device, and the measurement value indicated the presence or absence of the target SARS-CoV-2 RNA based on the Cutoff value (Figure 1B\&C). Figure 1D showed the appearance of the portable "suitcase laboratory" as alternative to large and precision equipment, which was able to give qualitative results in less than an hour undergoing only two steps, namely hybridization and immunofluorescence analysis (Figure 1E).

In this study, we chose to employ the data mode of T/C for the fluorescence signal to eliminate the background of difference test cards. By measuring the $\mathrm{T} / \mathrm{C}$ value of throat swab samples from 211 healthy population, the average background value was calculated to be 49.95 , with the standard deviation (S.D.) of 17.16 (Figure S1A). If the receiver operating characteristic (ROC) curve had been drawn for the test results of 100 clinical diagnosis cases (confirmed or excluded), the area under the curve (AUC) to evaluate the predictive accuracy was determined to be 0.999 with $95 \%$ confidence interval (CI) of 0.997-1.000 (Figure S1B). For the purpose of obtaining the highest sensitivity and specificity, the cutoff value was determined to be 102.07 corresponding to the Youden index point of 0.980 . For 100.00 was also much close to 2 times the average background 49.95 , T/C value of 100.00 was taken as the cutoff value for convenience in the following study.

\section{Optimizing of the SARS-CoV-2 specific DNA probes}

Optimizing DNA probes for the target RNA sequence of SARS-CoV-2 was the key factor to improve the efficiency of hybrid capture. All of DNA probe sequences we designed were shown in Table 1. The genome of SARS-CoV-2 is approximately 30,000 bases, including a variable number (6 to 11) of open reading frames (ORFs). The first ORF accounts for about $67 \%$ of the entire genome and encodes 16 non-structural proteins (nsp), and the remaining encodes helper proteins and structural proteins. The four main structural proteins are spike glycoprotein (S), small envelope protein (E), matrix protein (M), and nucleocapsid protein $(\mathrm{N})$ [12-13]. To date, the targeted genomic sites in SARS-CoV-2 nucleic acid detection mainly include three conserved regions in the viral genome, namely the open reading frame 1ab (ORF1ab), where the RNA-dependent polymerase gene (RdRp) is located [12], the Nucleocapsid protein $(\mathrm{N})$ gene and the envelope protein $(\mathrm{E})$ gene.

Table 1 Sequences of the DNA probes used in the HC-FIA detection.

\begin{tabular}{|c|c|c|c|}
\hline $\begin{array}{l}\text { Probe } \\
\text { Number }\end{array}$ & $\begin{array}{l}\text { Binding } \\
\text { region }\end{array}$ & $\begin{array}{c}\text { Sequence } \\
\left(5^{\prime}-3^{\prime}\right)\end{array}$ & $\begin{array}{c}\text { Sequence } \\
\text { length } \\
\text { (bp) }\end{array}$ \\
\hline cov01 & $\begin{array}{l}\text { ORF1ab } \\
\text { segment }\end{array}$ & AACGATTGTGCATCAGCTGACTGAAGCATGGGTTCGCGGAGTTGATCACAACTACAGCCATAACCTTTCCACATACCGCAGACGGTACAGACTGTGTTTTTAAGTGTAAAACCCACAG & 118 \\
\hline cov02 & $\begin{array}{l}\text { ORF1ab } \\
\text { segment }\end{array}$ & TCCTCACTGCCGTCTTGTTGACCAACAGTTTGTTGACTATCATCATCTAACCAATCTT & 58 \\
\hline cov03 & $\begin{array}{l}\text { ORF1ab } \\
\text { segment }\end{array}$ & СTTATCATCTTGTTTTCTCTGTTCAACTGAAGGTTTACTTTCAGTTATAAATGGC & 55 \\
\hline cov04 & $\begin{array}{c}\mathrm{N} \\
\text { segment }\end{array}$ & CAGACATTTTGCTCTCAAGCTGGTTCAATCTGTCAAGCAGCAGCAAAGCAAGAGCAGCATCACCGCCATTGCCAGCCATTCTAGCAGGAGAAGTTCCCC & 99 \\
\hline $\operatorname{cov} 05$ & $\begin{array}{c}\mathrm{N} \\
\text { segment }\end{array}$ & GCCTTTACCAGACATTTTGCTCTCAAGCTGGTTCAATCTGTCAAG & 45 \\
\hline $\operatorname{cov} 06$ & $\begin{array}{c}\mathrm{N} \\
\text { segment }\end{array}$ & CAGCAAAGCAAGAGCAGCATCACCGCCATTGCCAGCCATTCTAGCAGGAGAAGT & 54 \\
\hline cov07 & E segment & AAGCGCAGTAAGGATGGCTAGTGTAACTAGCAAGAATACCACGAAAG & 47 \\
\hline cov08 & E segment & CGAAGCGCAGTAAGGATGGCTAGTGTAACTAGCAAGAATACCACGAAAGCAAGAAAAAGAAGTACGCTATTAACTATTAACGTACCTGT & 89 \\
\hline cov09 & E segment & AGCAAGAAAAAGAAGTACGCTATTAACTATTAACGTACCTGT & 42 \\
\hline
\end{tabular}

We firstly found the RNA genome sequence of SARS-CoV-2 on the NCBI (National Center for Biotechnology Information) genebank, with the accession numbers: MN908947, MN908947.3, MN908947.2, NC_045512.1. Detailed analysis of other published sequences of SARS-CoV-2 genome revealed that no significant variation in these regions. With the aid of the design software Primer Premier 5.0, three probes were designed for each of the three regions. Among them, Cov01 and Cov04 located in the recommended region for the detection of the ORF1ab and N gene, respectively, announced by the Chinese Centers for Disease Control and Prevention (China CDC), while Cov08 accorded with the region of the E gene detection proposed by the literature [14] (Figure 1F).

Then the sequence alignment was conducted between the designed DNA probes and the human genome sequences, viruses, bacteria, mycoplasma, chlamydia, and other common pathogens. It only $100 \%$ matched the SARS-CoV-2 genomic sequence with no homology to human genomic DNA. Pseudoviruses carrying different segments of the target gene constructed using lentivirus as vectors were used as positive controls. The target gene sequences of pseudoviruses used was shown in Table S1. P1 was positive for the SARS-CoV-2 N segment, P2 was positive for the SARS-CoV-2 E segment, and P3 was SARS-CoV-2 ORF1ab segment was positive at a concentration of $3000 \mathrm{TU} / \mathrm{mL}$. Physiological saline, purified water and other common pathogens positive pharyngeal swab samples were used as negative controls N1-N17. The information of the positive and negative references used in the study was listed in Table S2. And the test results were shown as Table S3, S4 and S5. For ORF1ab segment, probes CoV01 and CoV03 were selected, and for E segment, probe CoV08 was selected. In the case of N segment, probe CoV04 and probe CoV06 were the preferred ones to bind target RNA. Then the 
selected DNA probes were further optimized by combination in the next step (Table S6), and each group of probes should simultaneously target all the three segments. HC-FIA test results in Table S7 showed that the probe combination of Cov01, Cov04, Cov08 (Group 2) not only successfully discriminated positive control samples from negative controls, but was capable of detecting low viral titer positive samples (1000 TU/mL) with a positive rate higher than 0.95. Therefore, Group 2 was selected out as the final combination.

\section{The specificity of HC-FIA}

After optimizing the probe sequences, we next examined the specificity of the HC-FIA method in detecting SARS-CoV-2. The positive controls included pseudoviruses (P1-P3) with target genes, and five clinical throat swab samples (P4-P8), which was determined by the nucleic acid detection kit (Shanghai ZJ Bio-Tech Co.,Ltd.). The negative controls were the confirmed influenza A, influenza B, respiratory syncytial virus, chlamydia pneumoniae, adenovirus or other pathogens positive and SARS-CoV-2 negative throat swab samples (N5-N17), and pseudovirus positive for the N segment of MERS (N18) or SARS coronavirus (N19), as listed in Table S2. The detection results of 8 positive reference samples and 15 negative reference samples were in line with expectations, as shown in Table S8.

We proceeded to investigate whether there were cross-reactions between 55 common pathogens related to respiratory diseases and SARS-CoV-2. The source and quantitative information of each pathogenic microorganism was provided in Table S9. The virus titer was determined to be $10^{6} \mathrm{pfu} / \mathrm{mL}$ by plaque assay, while for interference samples of bacteria, mycoplasma, and chlamydia, the concentration level was $10^{7} \mathrm{cfu} / \mathrm{mL}$. In addition, human genomic DNA was extracted and quantified to be $90-105 \mu \mathrm{g} / \mathrm{mL}$ from three whole blood samples. The results indicated that no significant cross reaction between all other pathogen samples (Table S10) or human genomic DNA (Table S11) and specific DNA probe sequences, and the HC-FIA method exhibited excellent specificity for SARS-CoV-2 detection.

\section{The sensitivity and precision of HC-FIA}

Furthermore, the pseudoviruses samples containing three sections of target genes were serially diluted to titers of 5000, 2500, $1000,800,500,250$ and 100 $\mathrm{TU} / \mathrm{mL}$ (absolutely quantified by digital PCR). Average the T/C values of 20 parallel tests. Figure 2A showed that the limit of detection (LOD) of SARS-CoV$2 \mathrm{~N}$ segment, E segment or ORF1ab segment positive pseudovirus was as low as $1000 \mathrm{TU} / \mathrm{mL}$ with positive rate greater or equal to $95 \%$, that is, 500 copies/mL as determined by digital PCR. Moreover, when the titers of pseudoviruses samples reached $10^{8} \mathrm{TU} / \mathrm{mL}$, no significant $\mathrm{HOOK}$ effect was observed (Figure S2). Simultaneously, it is indicated that the linear range of the assay located in the titer range from $10^{3}$ to $10^{6-7} \mathrm{TU} / \mathrm{mL}$.

Throat swab samples from 3 critical patients, proved to be positive by RT-qPCR and viral load further quantified based on digital PCR, were mixed with negative throat swabs samples to prepare serial dilutions of 2000, 1000, 500, 400 and 250 copies/mL. Similarly, the results showed that the LOD for clinical samples was revealed to be $500 \mathrm{copies} / \mathrm{mL}$ with positive rate greater or equal to $95 \%$ (Figure 2B). Moreover, the clinical throat swab samples close to the critical value of positive ones $(512,489$, and 497 copies/mL of the ORF1ab segment according to digital PCR) were employed to verify the LOD. Being performed 20 times in parallel, the positive rates of the critical samples were documented to be higher than or equal to $95 \%$.

To evaluate the performance of HC-FIA kit in SARS-CoV-2 detection for precision, 20 times of parallel tests for each clinical throat swab sample were performed in 5 consecutive days. And the representative samples we chose included a positive one (1348 copies / mL), a critical one (512 copies / mL) and a negative one ( 0 copies / mL). The average T/C values of the three batches in detecting the positive sample were as follows: $199.92 \pm 8.25$ (CV: 4.13\%), $200.68 \pm 7.91$ (CV: $3.94 \%)$ and $199.03 \pm 7.43$ (CV: 3.73\%). For the critical sample, they were $109.17 \pm 5.68$ (CV: $4.65 \%), 110.80 \pm 5.63$ (CV: $5.08 \%$ ), $111.48 \pm 4.67$ (CV: $4.19 \%)$, and for the negative one, $44.66 \pm 3.36$ (CV: $7.52 \%), 43.99 \pm 2.72$ (CV: $6.18 \%)$, and $44.72 \pm 2.98$ (CV: $6.66 \%)$. The batch-to-batch CV were $3.89 \%, 4.66 \%$, and $6.74 \%$, lower than $10 \%$. In summary, the above results indicated that the reagents and related products of HC-FIA exhibited good precision and reproducibility in detecting SARS-CoV-2.

\section{The robustness of HC-FIA}

In order to examine the robustness of HC-FIA, the effects of the endogenous interference substances, such as hemoglobin, mucin, and exogenous interference substances, namely common clinical drugs utilized in the treatment of patients with respiratory infection, including antiviral drugs, antibiotic and hormone, were systematically evaluated.

There were 18 clinical throat swab samples used for this experiment, including six critical samples (500-530 copies/mL for ORF1ab segment based on digital PCR), six negative samples, six positive samples. The T/C value of the previous interference-free test was the basic value, and the ratio of the later $\mathrm{T} / \mathrm{C}$ value with interference to the basic value was used to investigate whether the interference existed or not. In the prepared interference samples, the hemoglobin concentrations were $0.5,1.0$, and $2.0 \mathrm{~g} / \mathrm{L}$, and in the case of mucin, the concentrations were $5,10,20 \mathrm{~g} / \mathrm{L}$. The drug concentrations of exogenous interference samples were provided in Table S12-S14 according to the peak plasma concentration in vivo. As expected, all the ratios of

interference samples to the corresponding basic ones located in the range of 0.9 to 1.1 (Table S15-S17 for hemoglobin, Table S18-S20 for mucin and Table S21-23 for exogenous interference substances), indicating the SARS-CoV-2 HC-FIA detection reagent had excellent anti-interference property.

\section{The clinical evaluation of HC-FIA in SARS-CoV-2 detection}

In order to further evaluate the performance of HC-FIA detection reagents, a randomized double-blind clinical trial was performed by comparing it with RTqPCR or clinical diagnosis results in three independent medical institutions. The SARS-CoV-2 RT-qPCR detection kit produced by Shanghai ZJ Bio-Tech Co.,Ltd., which had been approved by NMPA and considered to be of better quality was chosen for contrast research. The clinical diagnosis results were 
provided by the designated hospitals of CIVID-19, determined from the CT images combined with clinical manifestations of the patients. A total of 734 samples provided by 670 subjects were tested in parallel, among which there were 593 throat swabs samples and 141 sputum samples. The case enrollment criteria referred to "Diagnosis and treatment of novel coronavirus infection pneumonia (Trail Version 6.0)" of China. The raw data of the clinical trial in Excel was provided as a separate supporting document.

Among the 670 cases enrolled in the trial, 313 cases were males, accounting for $46.72 \%$, and 357 cases were females, accounting for $53.28 \%$. As shown in Figure 3, the enrolled population was in line with demographic characteristics of SARS-CoV-2 infection according to different age groups [15]. Moreover, the visiting rate and diagnosis rate probably basically balanced with clinical actual conditions.

A total of 621 cases of HC-FIA test results were consistent with clinical diagnosis (Table 2). Among them, 210 cases were confirmed, and 411 cases were excluded. 49 cases were inconsistent with the clinical diagnosis results, of which 27 cases were confirmed, with the HC-FIA test results negative, and 22 cases were excluded, with the HC-FIA test results positive.

Table 2 Qualitative analysis of the 670 cases*.

\begin{tabular}{llccc}
\hline & & \multicolumn{3}{c}{ Clinical Diagnosis } \\
\cline { 3 - 5 } & & $\begin{array}{c}\text { Confirmed } \\
\text { case }\end{array}$ & $\begin{array}{c}\text { Excluded } \\
\text { Case }\end{array}$ & Total \\
\hline HC-FIA & Positive & 210 & 22 & 232 \\
& Negative & 27 & 411 & 438 \\
\cline { 2 - 5 } & Total & 237 & 433 & 670 \\
\hline
\end{tabular}

*Note[Each case was included only once. One case that simultaneously sampling sputum and throat swabs was only enrolled the sputum sample.

Table 3 Qualitative analysis of the 734 samples*.

\begin{tabular}{llccc}
\hline & & \multicolumn{3}{c}{ RT-qPCR } \\
\cline { 3 - 5 } & & Positive & Negative & Total \\
\hline HC-FIA & Positive & 249 & 4 & 253 \\
& Negative & 0 & 481 & 481 \\
& Total & 249 & 485 & 734 \\
\hline
\end{tabular}

*NotelAll tests of all cases are included in the table. If repeated measure was required, the retested results were enrolled in the group for analysis.

As shown in Table 3, a total of 730 samples of HC-FIA test was consistent with RT-qPCR results, of which 249 were positive and 481 were negative. There are 4 samples among them inconsistent with each other, namely, the HC-FIA test reagents made the judgement of positive while the RT-qPCR results were negative. Detailed analysis of the 4 samples are as follows: 3 of them were clinically diagnosed as COVID-19 excluded cases, and the RT-qPCR results agreed well with the clinical diagnosis, indicating the HC-FIA test gave false positive results. One sample was clinically diagnosed as a confirmed case, and herein the HC-FIA test was in accordance with the clinical diagnosis.

According to the data in Table 4, statistics analyses gave direct evidence that HC-FIA showed high agreement with clinical diagnosis, with the Kappa coefficient calculated to be 0.8393 , higher than 0.75 . For the contrast research of HC-FIA and RT-qPCR, similar conclusion was reached since the all Kappa coefficients obtained were higher than 0.98 , regardless of the sample type (Table 5).

In summary, our HC-FIA test kit was proved to have high-consistency with the clinical diagnosis results. Meanwhile, complete statistical analyses indicated the HC-FIA test kits had no statistical difference with the RT-qPCR products approved for marketing, namely the performances of the two test kits were equivalent for clinical application.

Table 4 Statistical analysis of HC-FIA and clinical diagnosis results for all cases.

\begin{tabular}{cccccccc}
\hline & \multicolumn{2}{c}{ Sensitivity } & Specificity & & \multicolumn{2}{c}{ Total data } \\
\hline & Consistency rate & $95 \%$ confidence interval & Consistency rate & $95 \%$ confidence interval & Consistency rate & $95 \%$ confidence interval & \\
\hline \multirow{2}{*}{ Throat swabs } & $87.69 \%$ & $83.08 \% \sim 92.30 \%$ & $95.01 \%$ & $92.82 \% \sim 97.20 \%$ & $92.53 \%$ & $90.38 \% \sim 94.68 \%$ \\
Sputum & $91.94 \%$ & $85.16 \% \sim 98.72 \%$ & $92.41 \%$ & $86.57 \% \sim 98.25 \%$ & $92.20 \%$ & $87.77 \% \sim 96.63 \%$ & 0.8419 \\
All samples & $88.61 \%$ & $84.57 \% \sim 92.65 \%$ & $94.92 \%$ & $92.85 \% \sim 96.99 \%$ & $92.69 \%$ & $90.72 \% \sim 94.66 \%$ & 0.8393 \\
\hline
\end{tabular}

Table 5 Statistical analysis of HC-FIA and RT-qPCR results for all samples.

\begin{tabular}{cccccccc}
\hline & \multicolumn{2}{c}{ Sensitivity } & Specificity & & \multicolumn{2}{c}{ Total data } \\
\hline & Consistency rate & $95 \%$ confidence interval & Consistency rate & $95 \%$ confidence interval & Consistency rate & $95 \%$ confidence interval & coeffiency \\
\hline Throat swabs & $100 \%$ & $99.85 \% \sim 100 \%$ & $99.26 \%$ & $98.43 \% \sim 100 \%$ & $99.49 \%$ & $98.92 \% \sim 100 \%$ & 0.9883 \\
Sputum & $100 \%$ & $99.74 \% \sim 100 \%$ & $98.73 \%$ & $96.26 \% \sim 100 \%$ & $99.29 \%$ & $97.90 \% \sim 100 \%$ & 0.9856 \\
All samples & $100 \%$ & $99.87 \% \sim 100 \%$ & $99.18 \%$ & $98.38 \% \sim 99.98 \%$ & $99.46 \%$ & $98.93 \% \sim 99.99 \%$ & 0.9879 \\
\hline
\end{tabular}

Page 5/11 


\section{Discussion}

Herein, we constructed the HC-FIA method with great simplicity and availability as alternative to the widely accepted RT-qPCR. The usage of the monoclonal antibody S9.6, which specially recognized DNA-RNA double-stranded hybrids, enabled the conversion of nucleic acid testing into immunofluorescence. In the DNA probe designation, we followed the general principles of PCR, such as the random distribution of bases, avoiding the generation of secondary structures and repeated DNA sequences and so on. It is noted that the probes here were 40-120 bp in length, much longer than general PCR primers and taqman probes. Furthermore, the selected DNA probes (CoV 01, 04 and 08) had the maximum length among them. This was due to the fact that long probes could facilitate the formation of longer hybrids, thereby increasing the sensitivity of recognization by S9.6 [3]. Therefore, although there was no nucleic acid amplification in HC-FIA, the LOD of HC-FIA was comparable to that of commercial RT-qPCR technology (2001000 copies $/ \mathrm{mL}$ ) [16]. The affinity of the $\mathrm{S} 9.6$ for DNA-RNA hybrids, the molar ratio of the monoclonal antibody to the hybrid, and the efficiency of signal amplification were of critical importance to determine the detection sensitivity of HC-FIA method. The molar ratio of S9.6 to the RNA-DNA hybrid was documented to be 11-13 under certain conditions, that was, a hybrid can bind to more than a dozen antibodies, leading to the signal amplification. The chromatographic process might have unavoidable effects on the absolute fluorescence values of the $\mathrm{T}$ line, as well as the $\mathrm{C}$ line, and in turn affected the stability of the results. Since the similar negative effect would be brought about to both the $\mathrm{T}$ line and the $\mathrm{C}$ line in the same test card, we read the $\mathrm{T} / \mathrm{C}$ value to improve the precision and reproducibility of the assay.

Table 6 The detailed comparison between commercially available SARS-CoV-2 nucleic acid detection kit and HC-FIA test kit.

\begin{tabular}{|c|c|c|}
\hline Pricinple & hybrid capture fluorescence immunoassay & Nuclein acid amplicaiton $n R T-q P C R \square$ \\
\hline Pre-treatment & Amplification-free & Nucleic acid extraction and purification \\
\hline Test time & Less than an hour & 3.5-4 hours \\
\hline Operating environment & Achieve on-site and on-demand detection, without the need for a PCR laboratory & $\begin{array}{l}\text { Require three professional PCR laboratories; if polluted, it cannot be used for a long } \\
\text { time }\end{array}$ \\
\hline $\begin{array}{l}\text { Requirements for } \\
\text { operator }\end{array}$ & No need for professional technicians, and operators can be trained simply & Require specially trained technicians \\
\hline Equipment & Supporting device, small and light, easy to carry & Precision instruments \\
\hline Throughput & 60 Tests per hour & 96 Tests $/ 4 \mathrm{~h}$ \\
\hline Application scenarios & $\begin{array}{l}\text { outpatient department, emergency department, customs and grassroots disease control } \\
\text { department etc. }\end{array}$ & Central laboratories of large hospitals \\
\hline Potential risk & No product to contaminate laboratory, and the reagent contains "anti-virus" component & Nucleic acid amplification will easily cause aerosol pollution, difficult to be \\
\hline pollution & & eliminated \\
\hline Transport and storage & At room temperature $\left(2-30^{\circ} \mathrm{C}\right)$ & Stored at $-20^{\circ} \mathrm{C}$, cold chain transport \\
\hline
\end{tabular}

Besides RT-qPCR, some isothermal amplification related SARS-CoV-2 nucleic acid detection assays were recently developed and also approved for listing. Our HC-FIA detection method saved the complicated nucleic acid extraction and reverses transcription processes. The entire detection process just involved two steps, namely nucleic acid hybridization and immunofluorescence analysis (Figure 1E and Table S24) greatly improved the efficiency of molecular diagnosis. The novel coronavirus had been inactivated in the step through incubating at $56{ }^{\circ} \mathrm{C}$ for 30 min (Table S24), and the detection process did not need to amplify the nucleic acids, which will not cause the aerosol pollution, thereby reducing the biosafety risk to environments. As shown in Table S24, the materials needed in each test cost only c.a. 2Х, lowering the applying threshold in developing countries. The portable fluorescence signal reading device made the method rely little on equipment and professional operator, meeting the demand of POCT and benefiting its application as on-site detection method for outpatient department, emergency department, customs and grassroots disease control department. The specific characteristics and advantages over RT-qPCR was listed as Table 6.

Of course, there was other similar visible lateral flow dipstick method called CRISPR diagnostics technology based on Cas 13 or Cas 12 presented by other researches [17-21]. However, nucleic acid extraction and recombinase polymerase amplification or loop-mediated amplification were still necessary in the procedure, raising the requirements for the testing environment, just as pre-amplification work area. Moreover, even though the whole process could be finished in an hour, as rapidly as HC-FIA, the LOD of it was reported to be $10^{4}-10^{5}$ copies $/ \mathrm{mL}$ for SARS-CoV-2 [17, 21] and 1000 copies/mL for Zika virus (ZIKV) and dengue virus (DENV) [19]. And large-sample clinical trials had not yet begun to verify their effectiveness and practicality. Altogether, HC-FIA based SARS-CoV-2 detection method was capable of serving as a sure safeguard in containing the epidemic through reducing community spread and imported cases from its root causes, especially in the developing countries.

\section{Conclusion}

In this paper, we presented a novel amplification-free POCT rapid SARS-CoV-2 nucleic acid detection system based on hybrid capture and immunofluorescence analysis, which included detection reagents, immunofluorescence lateral flow dipstick and a fluorescence analysis device. Optimized DNA probes targeting ORF1ab segment, $\mathrm{N}$ segment and E segment were documented to detect the pseudovirus containing each segment and clinical 
throat swab samples as low as 500 copies/mL, comparable to the commercial kits. And no significant cross-reaction was found between the SARS-CoV-2 probes and 55 common respiratory pathogens. The performance of the HC-FIA detection reagent would not be interfered with by the hemoglobin, mucin and various possible drugs that might exist in the sample. Clinical trials of HC-FIA test kit in 670 cases indicated that the rapid HC-FIA method (less than an hour) was capable of providing result identical to the one of approved RT-qPCR products and clinical diagnosis. Considering the HC-FIA test kit meets the demand of point-of-care technology (POCT), it will not only put forward the proper solution of rapid and high-throughput molecular diagnosis during the outbreak of SARS-CoV-2, but also pave the way for extending its application to other RNA virus detection.

\section{Methods}

\section{Antibodies}

The monoclonal antibody S9.6 (Cat. \# MABE1095, Lot \# 3275033) used in the work was purchased from EMD Millipore Corporation (USA). It was purified with protein $\mathrm{G}$ and was stored in buffer containing $0.1 \mathrm{M}$ Tris-Glycine $(\mathrm{pH} 7.4), 0.15 \mathrm{M} \mathrm{NaCl}$ with $0.05 \%$ sodium azide at $2-8^{\circ} \mathrm{C}$. Rabbit IgG $(\mathrm{Mw}=150 \mathrm{kDa}$, Product No. AGRIG-0100, Lot No. 021428216) and goat anti rabbit IgG (Mw=150kDa, Product No. ABGAR-0500, Lot No. 011530057) were purchased from Arista Biologicals Inc. (USA) and stored in $\mathrm{pH} 7.4$ phosphate buffered saline at $2-8^{\circ} \mathrm{C}$. The former was purified by protein $\mathrm{A}$ from normal rabbit serum (purity $\geq 90 \%$ ), while the latter was purified from whole goat antisera by immunoaffinity chromatography (purity $\geq 90 \%$ ).

\section{DNA probes and reagents}

The DNA probes were synthesized by Bioligo Inc. (Shanghai, China) and purified by means of high-performance liquid chromatography (purity $\geq 95 \%)$. N-(3dimethylaminopropyl)-N'-ethylcarbodiimide hydrochloride (EDC, CAS: 25953-53-8), hemoglobin (CAS: 9008-02-0) and mucin (CAS: 114956-81-9) was purchased from Sigma-Aldrich Chemical Co. (St. Louis, MO). Fluorescence nanoparticles (FNPs) composed by Europium chelate (diameter: 210 nm) with carboxylated surface were produced by Nanjing Microdetection Bio-Tech Co., Lt (Lot No.: MD20190831-1). Exogenous interference substances, namely drugs that might be used in treating respiratory infection patients were commercially available.

\section{Construction of pseudovirus}

The pseudovirus, used as positive or negative reference in the study, was performed by Sangon Biotech (Shanghai) Co. Ltd. Lentiviral vector system consisted of three plasmids of serial lentiviral vector (carrying target gene), psPAX2 vector and pMDA2G vector. Three plasmids were co-transfected into 293T cells using the calcium phosphate method. Target RNA was transcribed, and the proteins were translated from psPAX2 and pMD2G. Then the RNA and protein were packaged as lentiviruses. The information of the target gene sequences carried by pseudoviruses was listed in the Table S1. The titer of obtained pseudovirus particles was determined by fluorescence microscope counting of infected cells after being serial diluted.

The source and quantitative information of various pathogenic microorganisms employed in the cross-reaction testing was provided in Table S9.

\section{Labeling antibodies by FNPs}

$1 \%$ FNPs dispersing in deionized water $(1.0 \mathrm{mg} / \mathrm{mL})$ and EDC solution of $0.6 \mathrm{mg} / \mathrm{mL}$ were added to $0.05 \mathrm{~mol} / \mathrm{L} \mathrm{boracic} \mathrm{buffer}(\mathrm{pH}=8.0)$ on a rotary mixer for $20 \mathrm{~min}$. The FNPs were separated by centrifugation at 15000-16000rpm for 30min and resuspended in $0.05 \mathrm{~mol} / \mathrm{L}$ boracic acid buffer. Then the antibody solution of the final concentration $0.2 \mathrm{mg} / \mathrm{mL}$ was added to the activated FNPs solution, and the reaction system was placed on a rotary mixer for 2 hours. Add $0.1 \mathrm{ml} 10 \%$ BSA solution to $1.0 \mathrm{~mL}$ FNPs-labeled antibody solution for blocking, and place the mixture to a rotary mixer for $12-16$ hours. Finally, the FNPs-labeled antibody solution was separated through centrifugation at 15000-16000rpm, followed by being washed twice with an equal volume of $0.05 \mathrm{~mol} /$ Lboracic acid buffer, and the as-prepared mixture was resuspended in the washing buffer to obtain FNPs-labeled antibody solution.

\section{Fluorescence analysis device}

The supporting fluorescence analysis device (Registration Certificate No. 20172400062) was made by Anbio (Xiamen) Biotechnology Co.,Ltd, consisting of light source system, photoelectric detection system, signal amplifier circuit, thermal printer and touch screen.

\section{Operation procedure of HC-FIA Test kits}

The HC-FIA Test kits was produced by Anbio (Xiamen) Biotechnology Co.,Ltd. The operation process is strictly in accordance with the product manual. The operation procedure, approximate cost of the materials needed and duration of the HC-FIA assay was listed as Table S24. Firstly, prepare all reagents and specimens and equilibrate them to room temperature before use. Prepare reaction tube according to sample quantity, add $85 \mu \mathrm{L}$ of lysis buffer to every tube, shake the tube to mix the contents for next step. Add $20 \mu \mathrm{L}$ positive control, negative control and sample to the reaction tube. Then, reversal the control and sample tubes 3 times, incubate the tubes at $56^{\circ} \mathrm{C}$ in incubator for 30 minutes. Add $100 \mu \mathrm{L}$ of incubated sample to test card sample well and wait for 15 minutes. Insert the test card into the fluorescence analysis device, press "Start" and print the result. For quality control, before every new run of tests, the positive and negative control should give expected result to ensure that the test result is valid. If the control line " $\mathrm{C}$ " is invalid, there will be a remind text "Error" on analyzer, indicating that the result of the test is invalid.

\section{Clinical evaluation}

The randomized double-blind clinical evaluation of HC-FIA SARS-CoV-2 kits was performed independently in three independent designated hospitals of COVID-19. HC-FIA SARS-CoV-2 kits was provided by Anbio (Xiamen) Biotechnology Co.,Ltd. The RT-qPCR detection kit for contrast research were produced 
by Shanghai ZJ Bio-Tech Co.,Ltd and conducted on ABI 7500 Real-Time PCR System. This clinical trial was approved by the ethics committees for drug clinical trials of the three hospitals. The study was registered at http://www.chictr.org as ChiCTR2000033104. Because the samples used in the current trials was the remaining part after clinical nucleic acid detection, and double-blinded experimental design ensured the safety of patient privacy, the patients' informed consent was approved to be waived by the ethics committee. The case enrollment criteria referred to "Diagnosis and treatment of novel coronavirus infection pneumonia (Trail Version 6.0). Firstly, collect the clinical information of the enrolled cases, including: case number (ID number), age, gender, clinical diagnosis, sample collection time etc. The enrolled samples were randomly numbered by a random number generation tool, and then were arranged to test according to the random order. If the result shows the internal reference is invalid, repeat the test. A total of 734 samples of 670 subjects were evaluated in parallel, including 593 throat swabs samples of and 141 sputum samples. The related clinical diagnosis results were provided by corresponding hospital according to the results of CT images combined with clinical manifestation of the patients.

\section{Statistical analyses}

ROC curves and AUC value were employed to assess diagnostic accuracy of the HC-FIA method in determining the cutoff value using SPSS 19.0 software. For the consistency analysis between the HC-FIA and RT-qPCR or clinical diagnosis, Kappa statistics was used in the comparison. The Kappa coefficient less than 0.40 was supposed that the two methods were inconsistent with each other. The kappa coefficient in the range of $0.40-0.75$ was assumed moderate agreement, while the kappa coefficient greater than 0.75 indicating the high consistency. And two-tailed Mann-Whitney $U$ test was utilized to explore the significance of using Kappa coefficient. Part of the results in parallel experiments was presented as mean \pm standard deviation (S.D.).

\section{Declarations}

\section{Acknowledgments}

We thank Dr. Youqin Yan, Dr. Jianzhong Liu, Dr. Shimin Wu and Dr. Shiguo Liu for collecting samples and providing assistance in clinical trials. We appreciate Dr. Jidong Wang and Dr. Yulong Zhang for the help in plotting graphs. The work was funded by National Key Research and Development Project of China.

\section{Conflicts of interest}

There are no conflicts to declare.

\section{Author Contributions}

W. D. conceived the research, ran the experiments and prepared the manuscript. T. Y. supervised all aspects of the work and gave guidance. W. X. and H. S. helped design clinical trials, drafted the manuscript and contributed to data analysis. C. M and L. L. collected samples from patients and participated in the clinical evaluation. L. Y., Z. J., Z. L. and H. X. performed experiments, as well as clinical trial, and analyzed the raw data. Z. X. and B. J. participated in statistical analyses. S. M. provided professional advice on design of analysis device. All authors read, edited and approved the submitted manuscript.

\section{References}

1. Chen, L. et al\RNA based mNGS approach identifies a novel human coronavirus from two individual pneumonia cases in 2019 Wuhan outbreak. Emerg. Microbes. Infect. 9, 313-319 (2020)

2. Qiu J. Covert coronavirus infections could be seeding new outbreaks. Nature doi: 10.1038/d41586-020-00822-x (2020)

3. Boguslawski, S.J . et al. Characterization of monoclonal antibody to DNA • RNA and its application to immunodetection of hybrids. J. Immunol Methods. 89, 123-130 (1986)

4. Yan, Q., Shields, E. J., Bonasio, R. \& Sarma, K. Mapping native R-Loops genome-wide using a targeted nuclease approach. Cell Reports 29: 1369-1380 (2019)

5. Paull, T. T. RNA-DNA hybrids and the convergence with DNA repair. Crit. Rev. Biochem. Mol. 54, 371-384 (2019)

6. Toubiana, S. \& Selig, S. DNA:RNA hybrids at telomeres-when it is better to be out of the (R) loop. FEBS J. 285, 2552-2566 (2018)

7. Hu, Z., Zhang A. \& Storz G. An antibody-based microarray assay for small RNA detection. Nucleic Acid Res. 34, e52 (2006)

8. Sipova, H. et al. Surface plasmon resonance biosensor for rapid label-free detection of microribonucleic acid at subfemtomole level. Anal. Chem. 82, 10110-10115 (2010)

9. Qavi, A. J., Kindt, J. T., Gleeson, M. A., Bailey, R. C. Anti-DNA:RNA antibodies and silicon photonic microring resonators: increased sensitivity for multiplexed microRNA detection. Anal. Chem. 83, 5949-5956 (2011)

10. Tran, H. V., Piro, B., Reisberg, S., Duc, H. T. \& Pham, M. C. Antibodies directed to RNA/DNA hybrids: an electrochemical immunosensor for microRNAs detection using graphene-composite electrodes. Anal. Chem. 85, 8469-8474 (2013)

11. Sguassero, A. et al. A simple and universal enzyme-free approach for the detection of multiple microRNAs using a single nanostructured enhancer of surface plasmon resonance imaging. Anal. Bioanal. Chem. 411, 1873-1884. (2019)

12. Lu, R., et al. Genomic characterization and epidemiolo-gy of 2019 novel coronavirus: implications for virus origins and receptor binding. Lancet 20 : 30251-30258. DOI: 101016/S0140-6736 (2020) 
13. Chan, J. F., et al Genomic characterization of the 2019 novel human-pathogenic coronavirus isolated from a patient with a typical pneumonia after visiting Wuhan. Emerg. Microbes. Infect. 9(1): 221-236 (2020)

14. Corman, V. M., et. al. Detection of 2019 novel coronavirus (2019-nCoV) by real-time RT-PCR. Euro Surveill. 25(3). doi: 10.2807/15607917.ES.2020.25.3.2000045 (2020)

15. Zhao, J., et. al. Relationship between the ABO Blood Group and the COVID-19 Susceptibility. medRxiv, Doi: https://doi.org/10.1101/2020.03.11.20031096. (2020)

16. Wang, X. D., Shi, J., Ding, W. F. Jv, S. Q \& Zhao, J. H. Research state and and application of new 2019 novel coronavirus nucleic acid detection Chin. J. Clin. Lab. Sci. 38, 81-84 (2020)

17. Enabling coronavirus detection using CRISPR-Cas13: An open-access SHERLOCK research protocol. https://mcgovern.mit.edu/2020/02/14/enablingcoronavirus-detection-using-crispr-cas13-an-open-access-sherlock-research-protocol/ (2020)

18. Myhrvold, C., Freije, C. A. \& Gootenberg J. S. Field-deployable viral diagnostics using CRISPR-Cas13. Science 360, 444-448 (2018)

19. Freije, C. A. et al. Programmable Inhibition and Detection of RNA Viruses Using Cas13. Mol. Cell 76, 826-837 (2019)

20. Kellner, M. J., Koob, J. G., Gootenberg, J. S., Abudayyeh, O. O. \& Zhang, F. SHERLOCK: nucleic acid detection with CRISPR nucleases. Nat. Protoc. 14, 2986-3012 (2019)

21. Broughton. J. P. et al. CRISPR-Cas12-based detection of SARS-CoV-2. Nat. Biotechnol. https://doi.org/10.1038/s41587-020-0513-4. (2020)

\section{Figures}

A

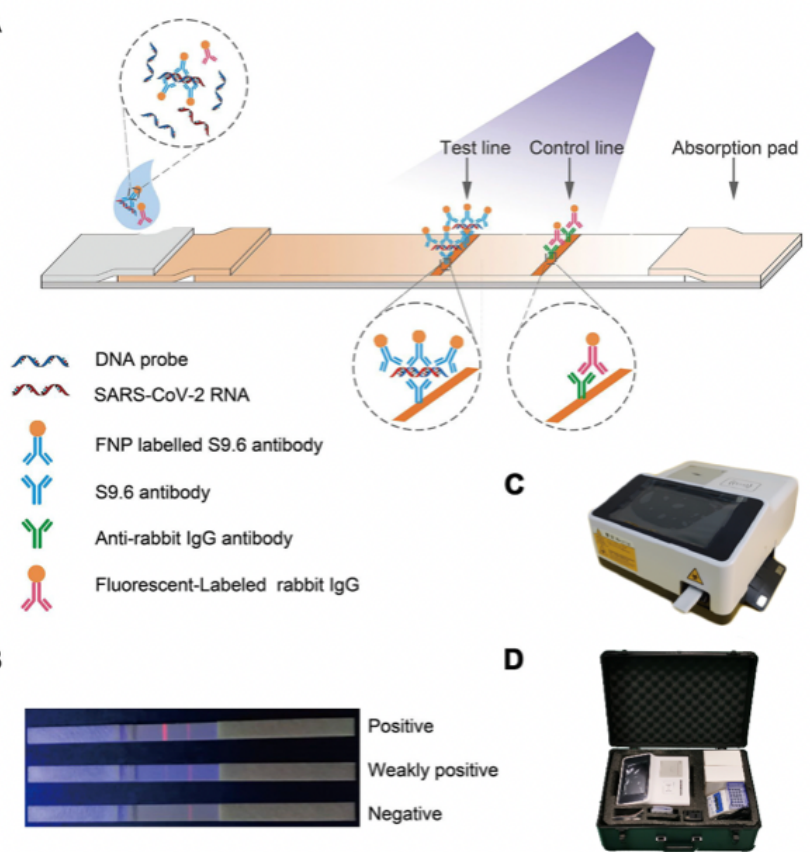

E

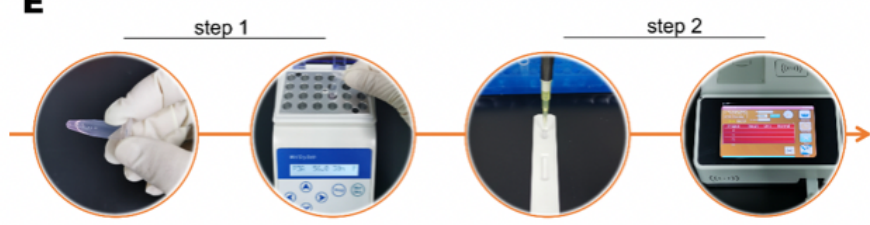

F

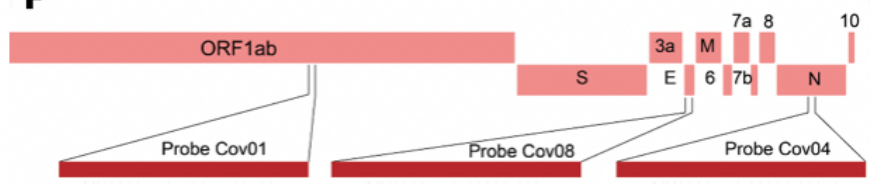

Figure 1

Schematic illustration of the SARS-CoV-2 HC-FIA detection system. (A) Principle of HC-FIA. (B) Representative results on lateral flow dipstick. (C) The fluorescence analysis device. (D) Appearance of the portable "suitcase laboratory". (E) The testing process of HC-FIA. Step 1 is nucleic acid hybridization in which a constant temperature incubator was needed. Step 2 means immunofluorescence analysis in which a miniaturized analysis device was employed to read the fluorescence signal of the test card. (F) The probe distribution in the genome map of SARS-CoV-2. 
A

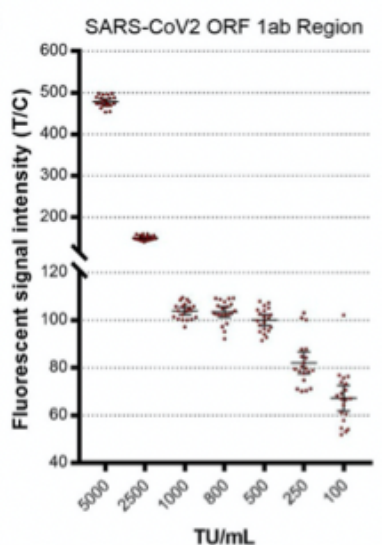

B

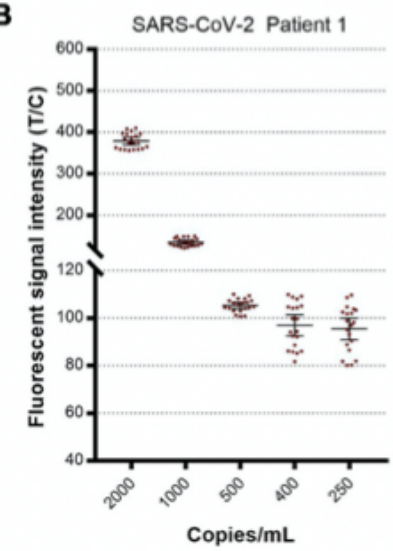

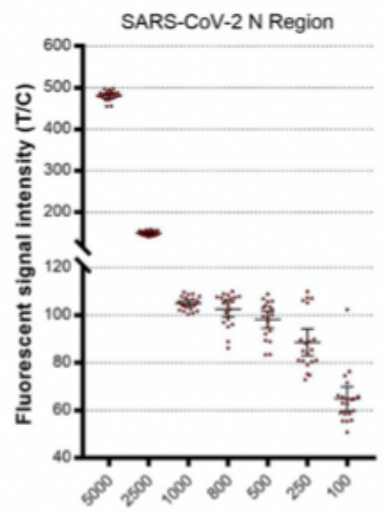

$\mathrm{TU} / \mathrm{mL}$

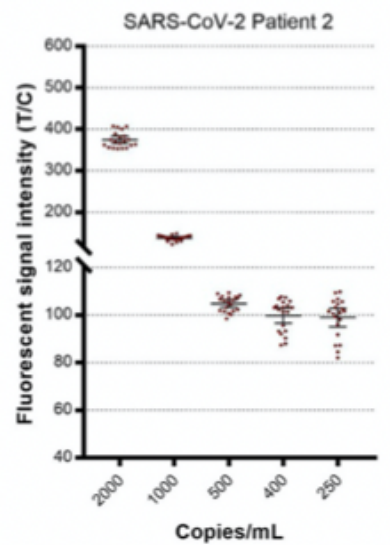

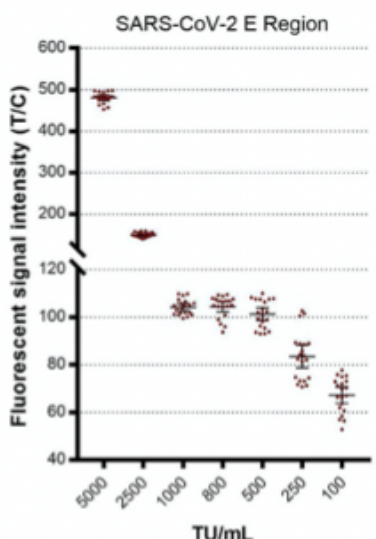

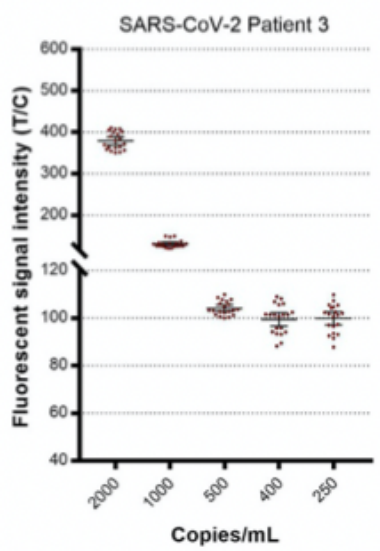

Figure 2

The sensitivity results of HC-FIA test. For each concentration, 20 tests were conducted in parallel. The LOD was determined as the concentration whose positive rate was greater or equal to $95 \%$ by HC-FIA test. (A) The data of HC-FIA test in detecting serially diluted SARS-CoV-2 N segment, E segment or ORF1ab segment positive pseudovirus. (B) The data of HC-FIA test in detecting serially diluted throat swab samples from 3 critical patients.

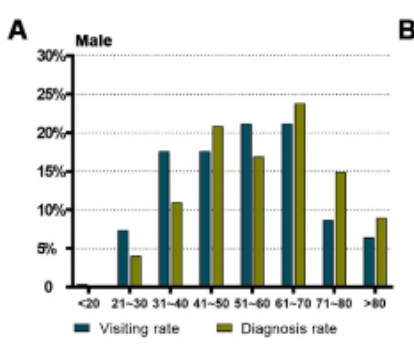

D

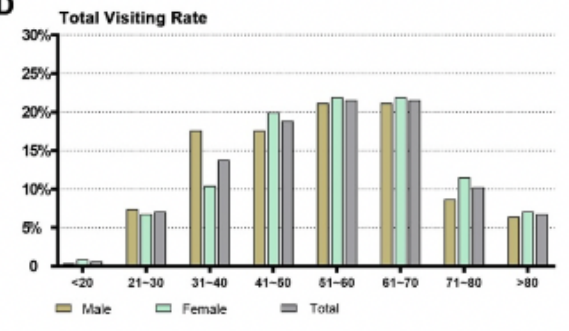

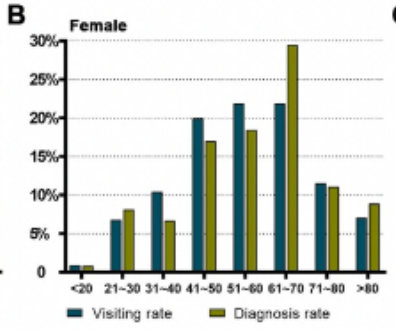

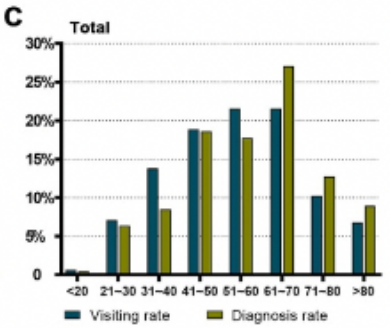

E

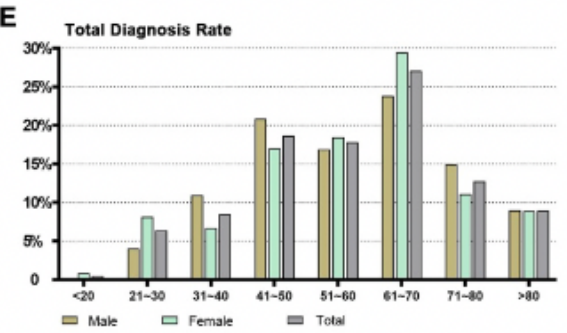

Figure 3

The visiting rate and diagnosis rate distribution in all enrolled cases broken down by age and sex determined from clinical diagnosis. The visiting rates were calculated by the number of visits of a certain age group divided by the total number of visits, in which confirmed and excluded cases were included. The diagnosis rate meant the percentage of the confirmed cases of a certain age range in all the confirmed patients enrolled. (A), (B) and (C) plotted the 
visiting and diagnosis rate according to different age groups and corresponded to man, woman and the total, respectively. (D) The illustration of visiting rate of different age groups. (E) The illustration of diagnosis rate of different age groups.

\section{Supplementary Files}

This is a list of supplementary files associated with this preprint. Click to download.

- DamingWangSupportinglnformation.pdf 